\title{
Diagnostic Value of Magnetic Resonance Image Feature Analysis under Reconstruction Algorithm for Knee Epiphyseal Injury
}

\author{
Ning He $(\mathbb{D}$, Changyou Tang $(\mathbb{D}$, Xianchao Zhou $(\mathbb{D}$, and Shuolin Feng $(\mathbb{D}$ \\ Department of Orthopedics, The Fourth Hospital of Changsha City, Changsha 410006, Hunan, China \\ Correspondence should be addressed to Ning He; 201801010323@stu.hnie.edu.cn
}

Received 15 October 2021; Revised 22 December 2021; Accepted 28 December 2021; Published 25 January 2022

Academic Editor: M Pallikonda Rajasekaran

Copyright ( $\odot 2022$ Ning He et al. This is an open access article distributed under the Creative Commons Attribution License, which permits unrestricted use, distribution, and reproduction in any medium, provided the original work is properly cited.

\begin{abstract}
To analyze the diagnostic value of magnetic resonance imaging (MRI) in epiphyseal injury of adolescent children, MRI images of 26 adolescent knee joint injuries were selected. The image display before and after reconstruction based on the compressed sensing theory (CS) algorithm was compared and analyzed, so did the signal-to-noise ratio of knee MRI reconstructed images under 2D random sampling and radial sampling at different sampling rates. The results showed that the sharpness, specificity, and accuracy of images reconstructed by the CS algorithm were significantly higher than those before reconstruction $(P<0.05)$. When the sampling rates were almost the same $(20 \%, 40 \%$, and $60 \%)$, the signal-to-noise ratio (SNR) of the reconstructed knee MRI images by random sampling was higher than that by radial sampling (about $9 \%, 6 \%$, and $3 \%$ higher, respectively), that is, the information contained by random sampling was larger than that by radial sampling. It overcame the defects of undisplaced epiphyseal injuries in children and adolescents that cannot be found by plain X-ray films and can clearly and accurately diagnose epiphyseal injuries and fracture types that cannot be seen by plain X-ray films. To sum up, the MRI image reconstruction algorithm based on compressed sensing theory can effectively improve the diagnosis effect of knee epiphyseal injury.
\end{abstract}

\section{Introduction}

Epiphysis, located at both ends of the immature bone, is made of cartilage and is particularly vulnerable to damage during childhood growth and development [1-3]. Therefore, if it is not treated in time after being damaged, it is likely to cause complications such as myositis ossificans, bone nonunion, angular malformation, knee joint adhesion, and infection [4]. Then, abnormal bone growth and development is caused, which significantly affects the physical and mental health of children. The key to improving the prognosis lies in the early diagnosis and timely and effective treatment of epiphyseal injury [5].

Clinical imaging examination is the main way to evaluate the degree of epiphyseal injury in adolescents and children, including X-ray plain film examination, ultrasound examination, CT examination, and MRI examination [6]. X-ray plain film is the first choice for examination of bone trauma, but due to incomplete epiphyseal ossification, X-ray plain film cannot develop normally, so that missed diagnosis and misdiagnosis often occur. Ultrasound is also widely used in the diagnosis of musculoskeletal diseases in children and adolescents because of its advantages of being fast and radiation-free. However, for older children with osteochondral structure becoming more mature, ultrasound is limited in the range of display by bone shadow, resulting in the overlapping phenomenon and affecting the diagnosis results $[7,8]$. CT examination has a good display effect on fracture fragments and has a good guiding role in preoperative evaluation and planning. However, for the epiphyseal plate, a plain CT scan can hardly show the direction of the fracture line in its axial image, so spiral CT was proposed. It can carry out continuous tomography imaging in sagittal and coronal positions of bone and joint, which successfully overcomes the disadvantages of X-ray plain imaging showing overlapping and CT plain scanning axial imaging, saving 
examination time. However, due to its high cost and strong radiation, its clinical promotion is limited [9]. MRI examination can clearly and accurately show the osteochondral, soft tissue, and bone condition in epiphyseal injury as well as the fissure trend of fracture line in epiphyseal. Its multidirectional imaging can show the complex anatomical structure of the knee joint from multiple angles and can also show the injury of the meniscus and ligament of the knee joint, which cannot be found by plain X-ray and CT. However, in the process of MRI scanning imaging, it takes too long, which reduces the utilization rate of the equipment. At the same time, it also has a certain impact on patients' physical and mental tolerance, which leads to the appearance of imaging artifacts, thus affecting clinical diagnosis and treatment $[10,11]$.

With the continuous development of science and technology and digital model, a breakthrough has been made in the signal processing field, especially the birth of compressed sensing theory (CS). Its emergence in the medicalrelated field (CT, medical imaging, MRI, etc.) has brought a great sensation. Relevant studies confirmed that, regarding MRI, the CS theory can reconstruct the original image in a very short time, and the design of $k$-space data collected by random measurement matrix and the construction of nonlinear algorithm combined with relevant mathematical knowledge can make high-quality image reconstruction. It uses the prior knowledge that signals meet sparsity to project the high-dimensional signals randomly into the low-dimensional space so that the projected data can accurately recover the original signals. If CS is used to realize signal reconstruction, the signal must meet the following conditions, that is, it is sparse itself or sparse in a transform domain. In the medical field, most of the internal tissue features and organ boundaries in MRI are sparse, which makes it possible to apply CS in medical image reconstruction $[12,13]$. With the advantages of CS theory, the MRI image reconstruction was carried out to analyze the diagnostic value of MRI image characteristics for adolescent children's knee epiphyseal injury in this research.

\section{Materials and Methods}

2.1. General Information. In this research, 26 patients with knee joint injury (including 18 males and 8 females) admitted to the hospital for examination from January 2020 to
December 2020 were selected as the research objects. The inclusion criteria were patients without closure of the epiphyseal line, aged between 8 and 18 years, with a mean age of 15 years. MRI was performed on all patients within a month of trauma, with a mean time of 7.5 days. Clinical manifestations were as follows: a total of 15 patients had limited knee movement, and 14 patients had soft tissue swelling around the knee. All patients had pain after trauma. The knee hyperextension test was positive in 11 cases, and the knee cyclotron compression test was positive in 7 cases. A total of 6 cases were positive in the Lachman test, 5 cases were positive in the knee flexion test, 4 cases were positive in the knee valgus stress test, and 3 cases were positive in drawer test after the $90^{\circ}$ bend. The patients and their families have fully understood the situation and signed the informed consent, and this study had been approved by the ethics committee of the hospital.

2.2. MRI Examination. $1.5 \mathrm{~T}$ superconducting MRI system was used to perform routine scans on the patient (in supine position, knee joint surface coil). Scanning methods of four sequences were coronal T1WI (TR300-600 ms, TE8-15 ms), spatial presaturated fat suppression proton density weighting (FS-PDWI) (TR2000-6000 ms, TE8-15 ms), sagittal T1WI (TR300-600 ms, TE8-15 ms), and spatially presaturated fat suppression proton density weighting (FSPDWI) (TR2000-6000 ms, TE8-15 ms). The coronal and sagittal layer thicknesses were both $4-5 \mathrm{~mm}$, the layer spacing was $1-1.5 \mathrm{~mm}$, and the FOV was $16-20 \mathrm{~cm}$. Four patients were scanned in the horizontal axis T2WI spatial presaturated fat suppression sequence (TR2000-6000 ms, TE80-150 ms), the layer thickness was $4 \mathrm{~mm}$, the layer spacing was $1 \mathrm{~mm}$, and the $\mathrm{FOV}$ was $18 \mathrm{~cm}$.

2.3. Principles of MRI. In the field of MRI in the imaging industry, the data space is called $k$-space, that is, the Fourier frequency space. Each MRI image will have unique $k$-space data corresponding to it [14]. The $K m$ and $K n$ directions of the $k$-space represent the frequency encoding direction and the phase encoding direction, respectively, and the gray value of each pixel on them determines the intensity of the magnetic resonance signal at that point. The gray value of any pixel in $k$-space is calculated by the following equation:

$$
S(K m, K n)=\iint \rho(m, n) e^{-i \cdot 2 \pi(m K m+n K n)} \mathrm{d} m \mathrm{~d} n, K m=\gamma \int_{0}^{t m} G m(t) \mathrm{d} t, K n=\gamma \int_{0}^{t_{n}} G n(t) \mathrm{d} t
$$

where $\rho(m, n)$ is the hydrogen proton density distribution corresponding to this pixel $(m, n)$.

MRI image reconstruction is continuously updating the $\mathrm{k}$-space data through a certain method and then passing the $\mathrm{k}$-space data through the inverse Fourier transform to obtain the desired MRI image. The equation of the two-dimensional inverse Fourier transform is shown in equation (2), and the schematic diagram of image reconstruction is shown in Figure 1.

$$
\rho(m, n)=\iint S(K m, K n) e^{i \cdot 2 \pi(m K m+n K n)} \mathrm{d} K m \mathrm{~d} K n .
$$

In practical applications, MRI image reconstruction uses existing knowledge and theories to find a robust algorithm to 


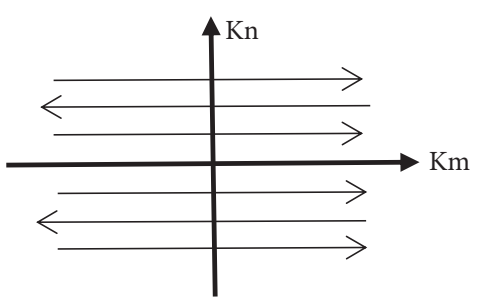

K-space

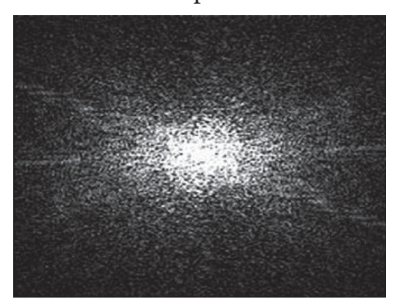

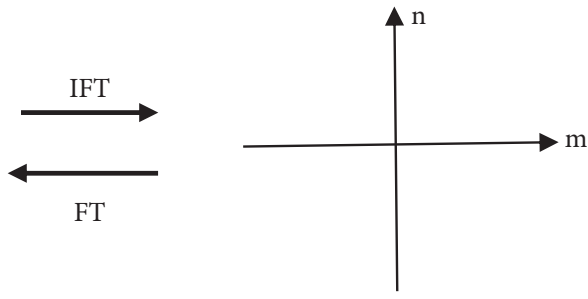

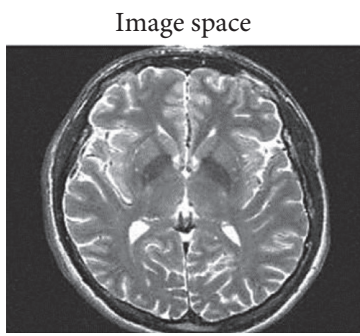

FIgURE 1: Schematic diagram of MRI image reconstruction through k-space.

reconstruct the original image more accurately from a small amount of $\mathrm{k}$-space data. Facts have shown that it is very important to reduce the scan time as much as possible, that is, to reduce the MRI reconstruction time as much as possible, which means, while keeping the quality of the reconstructed image unchanged, it is necessary to reduce the observation data as much as possible. The application of compressed sensing theory can greatly reduce the sampling rate of the signal and reduce the transmission of data, subsequent processing, and storage. Based on these characteristics, the application of compressed sensing theory in medical image reconstruction can be well applied to medical imaging that requires speed and radiation dose. Therefore, one of the more popular studies in the medical field is the application of compressed sensing.

In Figure 1, the amplitude diagram of the $k$-space is on the left, and the phase diagram of the $k$-space is on the right, which constitutes a complete $k$-space. $K_{\mathrm{m}}$ represents the frequency encoding direction, $K_{\mathrm{n}}$ represents the phase encoding direction, and IFT and FT represent the two Fourier transforms.

2.4. MRI Image Reconstruction Based on Compressed Sensing. At present, when compressed sensing was adopted for MRI image reconstruction [15], the most used objective function is as follows:

$$
\widehat{m}=\arg \min _{m} \frac{1}{2}\|R m-n\|_{2}^{2}+\alpha\|m\|_{T V}+\beta\|\psi m\|_{1} .
$$

In the equation, $R$ is the local Fourier transform operator. $m$ is the reconstructed target image. $n$ is the observation data of the MRI image, that is, the local Fourier transform data. $\Psi$ is the wavelet transform operator. A and $\beta$ are nonnegative parameters that weigh the effects of two sparse regularization terms in the objective function.

The use of total variation as the constraint regular term in equation (3) means that the image $m$ has a smaller total variation, and its definition is generally in the form of $\|m\|_{T V}=\sum_{i j}\left(\left(\boldsymbol{\Delta}_{1} m_{i j}\right)+\left(\boldsymbol{\Delta}_{2} m_{i j}\right)\right)$, where $\boldsymbol{\Delta}_{1}$ and $\boldsymbol{\Delta}_{2}$ are the difference operations in the horizontal and vertical directions, respectively. This form is the earliest proposed by $\mathrm{He}$ et al. and Lusting et al. They proposed this model on the basis that the MRI images of general organs are segmented and smooth, which means that there is a small total variation, and are also sparse in the wavelet transform domain, so this model is reasonable for MRI reconstruction. Many experiments have also proved that this model is better than using wavelet transform alone or full variation regular term reconstruction [16]. However, since $l_{1}$ and TV norms are convex and nonsmooth, it is very difficult to solve this problem. The conjugate gradient method (CG) and partial differential equation (PDE) proposed by He et al. (2018) [17] and Zhu et al. (2019) [18] can use equation (3) as the objective function to reconstruct MRI images. However, the calculation speed is the bottleneck of these two methods, thus inhibiting the effect of equation (3).

Pant and Krishnan (2014) [19] used the lp pseudonorm $(P<1)$ as the regular term and used the FOCUSS [20] algorithm to minimize the lp norm. Compared with the norm of $\mathrm{P}=1$, it can obtain a higher compression ratio [21, 22]. However, because the lp norm is nonconvex, the global optimal solution is usually not obtained. In response to these existing problems, scholars proposed the operator splitting algorithm (TVCMRI) [23] and the variable splitting algorithm (Recpf) [24] to solve the optimization problem (5). Compared with other algorithms, these two algorithms have faster speed and higher accuracy. Based on these algorithms, some researchers put forward a fast composite splitting algorithm (FCSA). This algorithm combines the advantages of the operator splitting algorithm and variable splitting algorithm and makes full use of the fast-iterative thresholding algorithm (FISTA) in the process of solving convexity problems. The corresponding experiments also proved that the algorithm is superior to the previously proposed algorithm in both speed and accuracy $[25,26]$. 


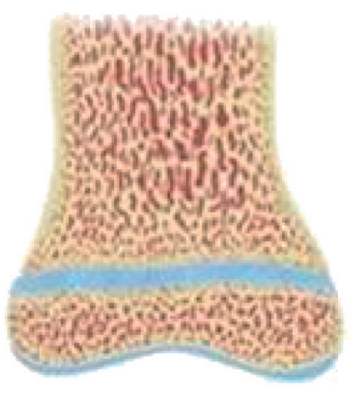

Normal

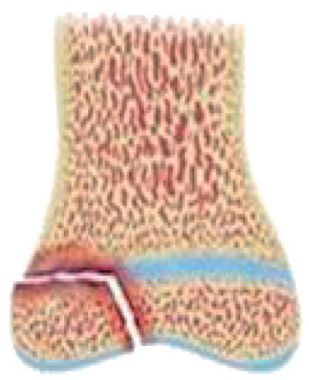

Type 3

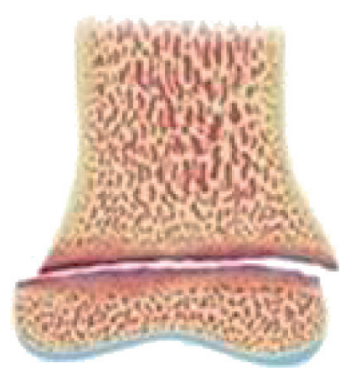

Type 1

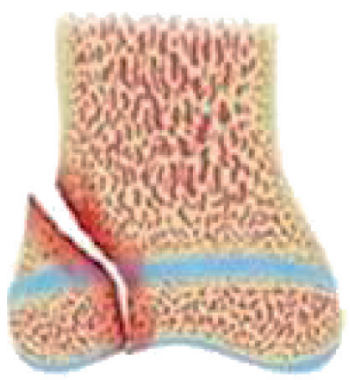

Type 4

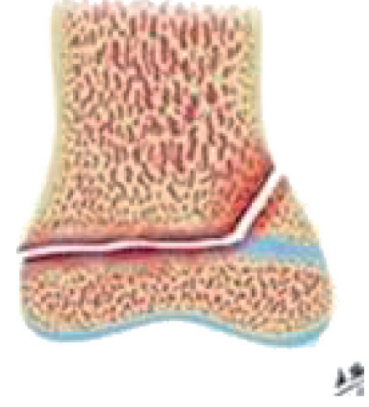

Type 2

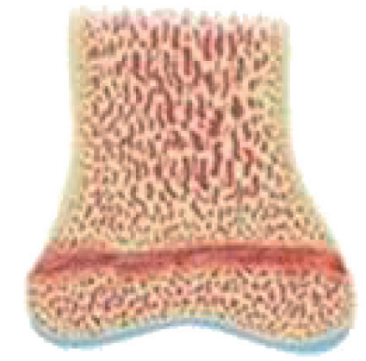

Type 5

FIGURE 2: Salter-Harris classification results of epiphyseal fractures.

2.5. Image Evaluation and Data Measurement. Clinical classification of fracture injuries in the epiphyseal region of children adopted the Salter-Harris classification [27], as shown in Figure 2.

(i) Type I: epiphyseal separation, fracture line only through the epiphyseal plate cartilage

(ii) Type II: common, accounting for $75 \%$ of epiphyseal plate fractures; fracture line through the epiphysis plate and extends to the epiphysis.

(iii) Type III: fracture line through epiphysis-to-epiphysis plate cartilage, but does not involve the epiphysis, that is, bone marrow part fracture, separation, no bone marrow end fracture, separated bone marrow slight displacement

(iv) Type IV: fracture line from the epiphysis through the epiphysis plate into the epiphysis, which is the easiest to cause early closure of epiphysis and angular deformity

(v) Type V: rare (epiphyseal plate injury less than 1\%), complete, or partial compression of the epiphyseal plate

(vi) Type VI: confined to the epiphysis and not involving the growth plate of the epiphysis is type VI (including intramedullary fracture involving the bone marrow cartilage and bone contusion without fracture line)

MRI images of 26 patients were observed by experienced diagnostic imaging physicians in a double-blind manner. The content included whether there was cartilage injury in the proximal tibia, distal femur, and patella, morphology, signal, width of damaged cartilage plate, and width of fracture line; whether there was epiphyseal fracture, the type of fracture, the shape, shape and width of fracture line; whether there was physeal contusion; whether there was injury or tear of the cruciate ligament and collateral ligament; whether the meniscus was damaged or torn; whether there was effusion in the knee cavity and suprapatellar sac; and whether there was any soft tissue damage.

\section{Results}

Of the 26 patients with epiphyseal injury, including 3 cases of Salter-Harris type I, all had distal epiphyseal fracture, partial widening and fracture of the epiphyseal plate, and meniscus tear. There were 5 cases of type II fracture, including 3 cases of distal femoral epiphyseal fracture and 2 cases of proximal tibial epiphyseal fracture. The fracture line extended through the epiphyseal plate to the epiphyseal. There were 7 cases of type III fracture, including 2 cases of distal femur epiphysis fracture, 3 cases of proximal tibia epiphysis fracture, and 2 cases of a patella avulsion fracture. The fracture line was from the articular surface through the epiphysis plate cartilage layer. Three cases of type IV fracture were all proximal tibial epiphyseal fractures, and the fracture line was from epiphyseal through the epiphyseal plate cartilage into the epiphyseal. One patient in type $\mathrm{V}$ had compression of the epiphyseal plate, and seven patients in type VI had an epiphyseal injury. There were 5 cases of distal femoral epiphyseal injury and 2 cases of proximal tibial epiphyseal injury, which were confined to the epiphyseal and did not involve the epiphyseal plate, as shown in Table 1 . The contrast images of MRI images before and after reconstruction under CS theory of some patients are shown in Figures 3 and 4 . 
TABle 1: The patient's epiphyseal injuries.

\begin{tabular}{|c|c|c|}
\hline $\begin{array}{l}\text { Salter-Harris } \\
\text { typing }\end{array}$ & Cases & Main manifestations \\
\hline Type I & 3 & Distal epiphyseal fracture, partial widening and fracture of the epiphyseal plate, and meniscus tear. \\
\hline Type II & 5 & $\begin{array}{l}3 \text { cases of distal femoral epiphyseal fracture and } 2 \text { cases of proximal tibial epiphyseal fracture, and the fracture } \\
\text { line extended through the epiphyseal plate to the epiphyseal. }\end{array}$ \\
\hline Type III & 7 & $\begin{array}{l}2 \text { cases of distal femur epiphysis fracture, } 3 \text { cases of proximal tibia epiphysis fracture, and } 2 \text { cases of a patella } \\
\text { avulsion fracture. The fracture line was from the articular surface through the epiphysis plate cartilage layer. }\end{array}$ \\
\hline Type IV & 3 & $\begin{array}{c}\text { Proximal tibial epiphyseal fracture, and the fracture line was from epiphyseal through the epiphyseal plate } \\
\text { cartilage into the epiphyseal. }\end{array}$ \\
\hline Type V & 1 & Epiphyseal plate compression. \\
\hline Type VI & 7 & $\begin{array}{c}5 \text { cases of distal femoral epiphyseal injury and } 2 \text { cases of proximal tibial epiphyseal injury, which were confined } \\
\text { to the epiphyseal and did not involve the epiphyseal plate. }\end{array}$ \\
\hline
\end{tabular}

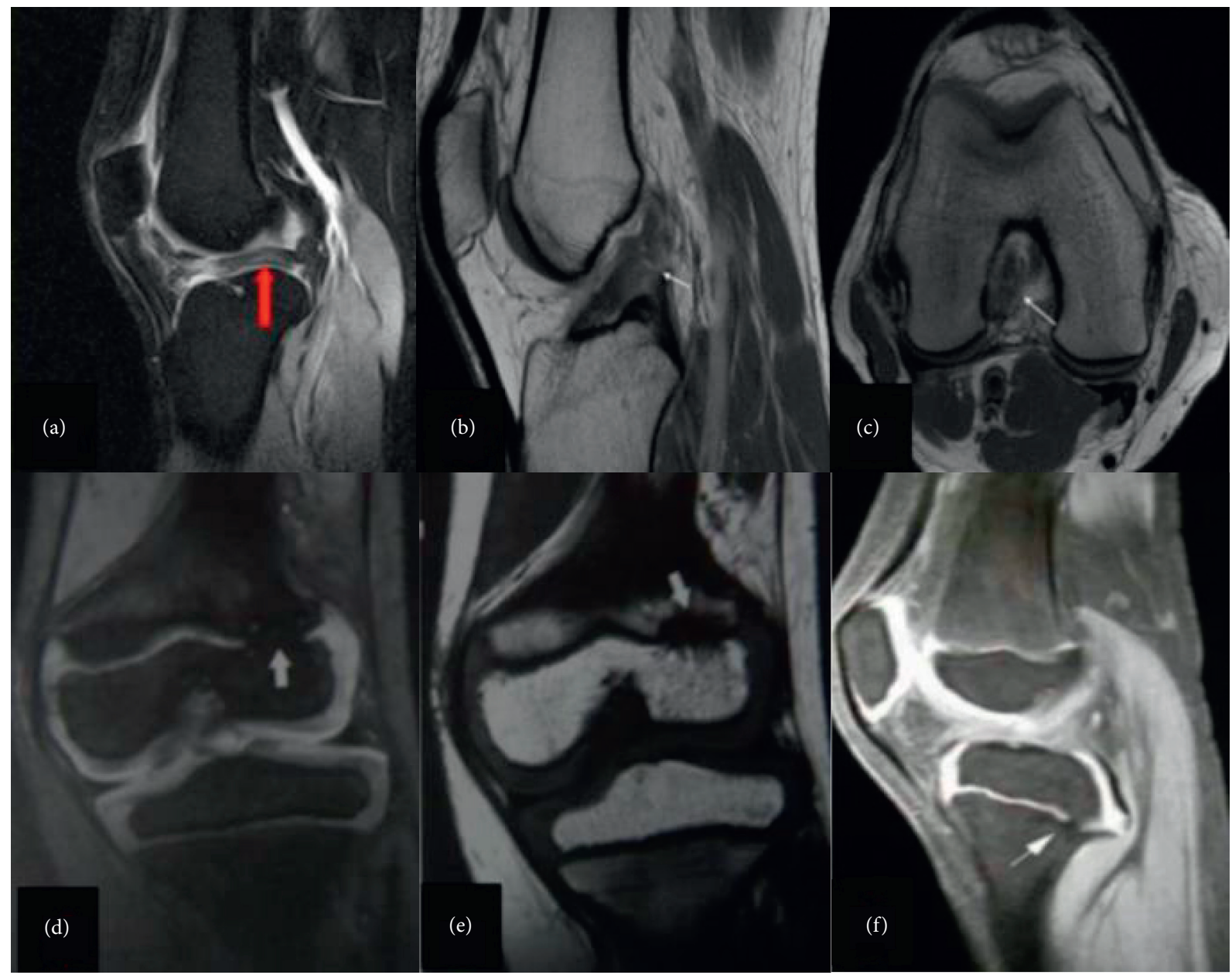

FIgURE 3: MRI image before reconstruction under compressed sensing theory. (a) A Salter-Harris type I patient, ((b), (c)) a Salter-Harris type II patient, ((d), (e)) a Salter-Harris type V patient, and (f) a Salter-Harris type VI patient. The arrow pointed to the site of injury.

At present, the sampling modes of MRI reconstruction include radial sampling, spiral variable density sampling, Cartesian sampling, and two-dimensional random sampling. In the experiment, two-dimensional random sampling, which can well retain useful information of the image, and radial sampling, which is relatively extensive in practical applications, were used. In these two sampling methods, different sampling rates were set, and the reconstruction algorithms under compressed sensing were compared. The MRI reconstructed images under two-dimensional random sampling and radial sampling are shown in Figures 5 and 6.

Figure 7 shows that when the sampling rate of the $2 \mathrm{D}$ random sampling method was $20.52 \%$, the signal-to-noise ratio of MRI images before and after reconstruction was $17.53 \%$ and $22.73 \%$, respectively. When the sampling rate was $40.31 \%$, the signal-to-noise ratio before and after MRI 


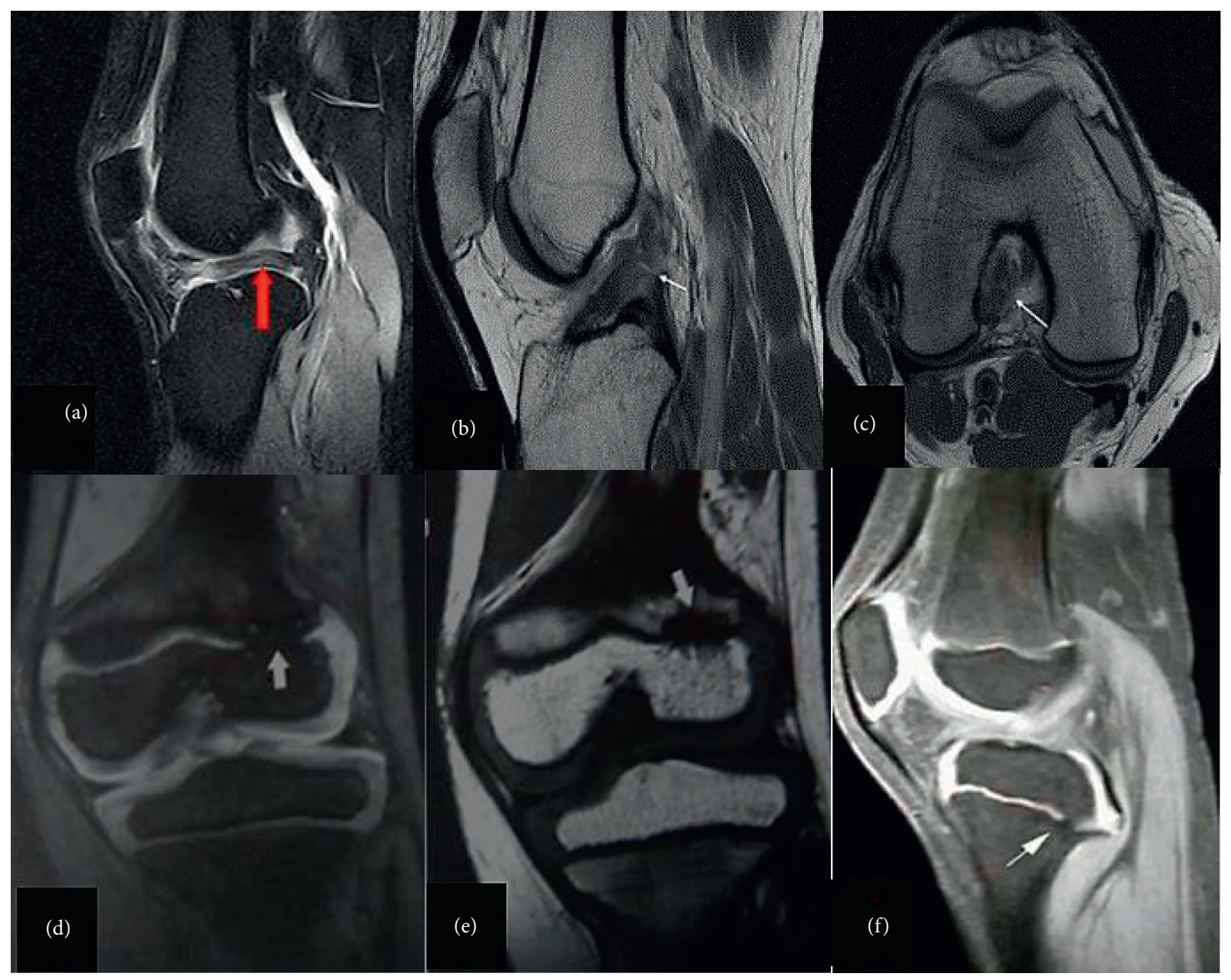

Figure 4: MRI image reconstruction under compressed sensing theory. (a) A Salter-Harris type I patient, ((b), (c)) a Salter-Harris type II patient, ((d), (e)) a Salter-Harris type V patient, and (f) a Salter-Harris type VI patient. The arrow pointed to the site of injury.
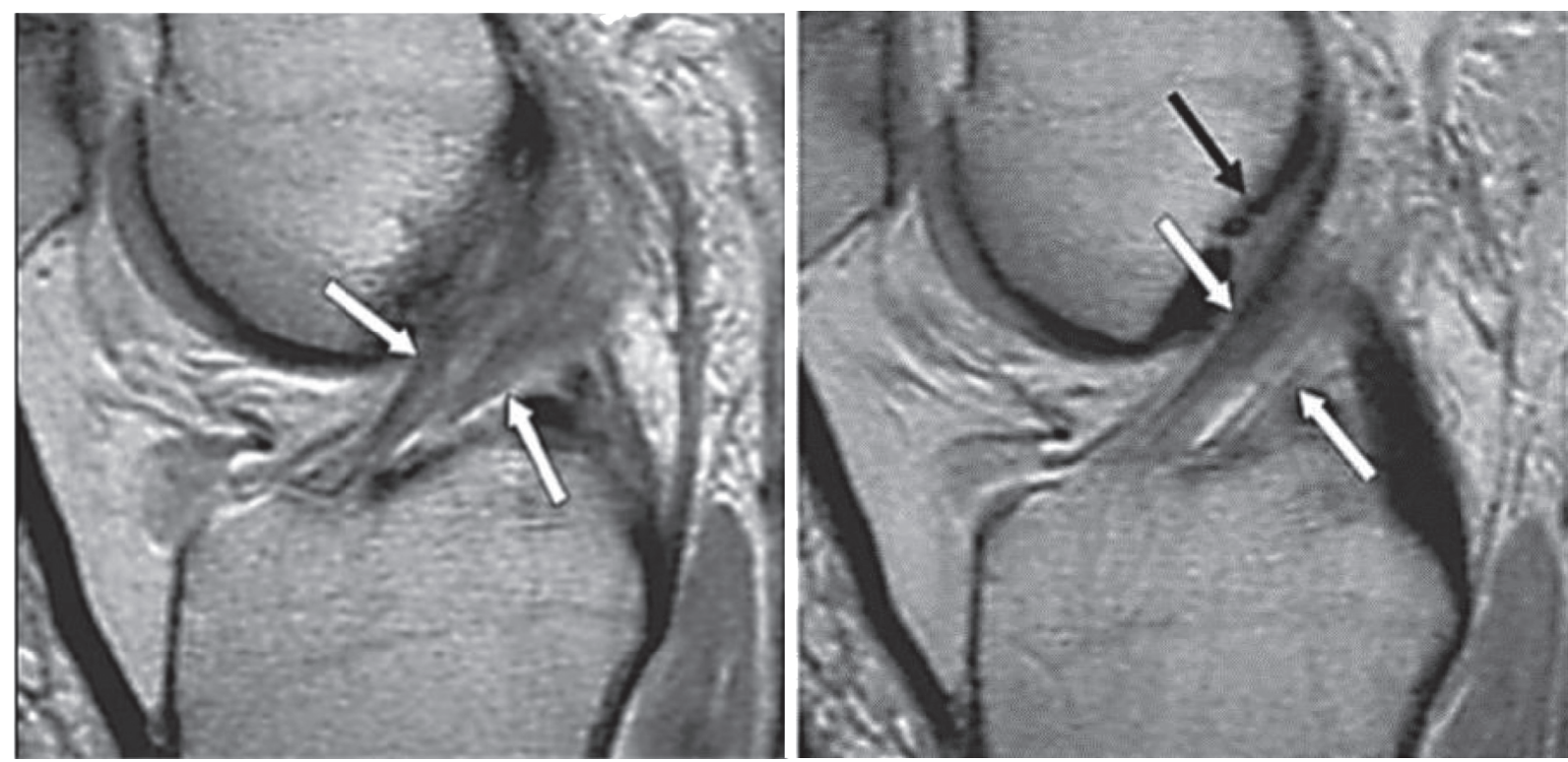

FIGURE 5: MRI reconstruction image under two-dimensional random sampling anterior cruciate ligament injury. The arrow pointed to the site of injury. 

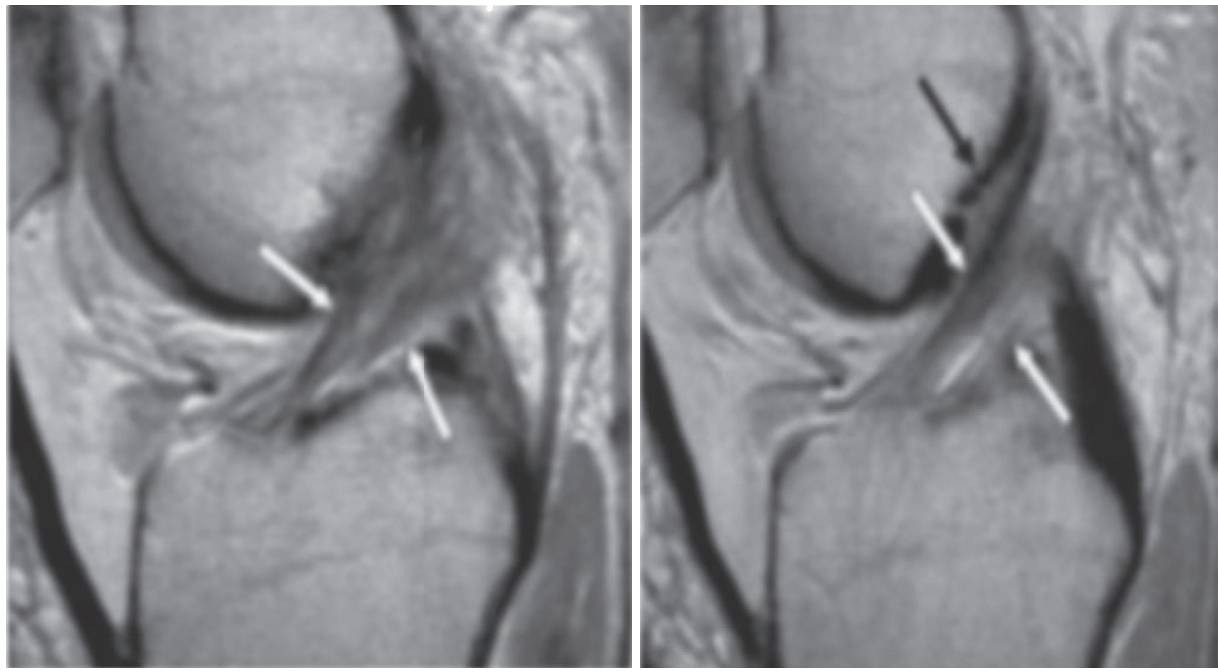

FIGURE 6: MRI reconstruction image under radial sampling anterior cruciate ligament injury. The arrow pointed to the site of injury.

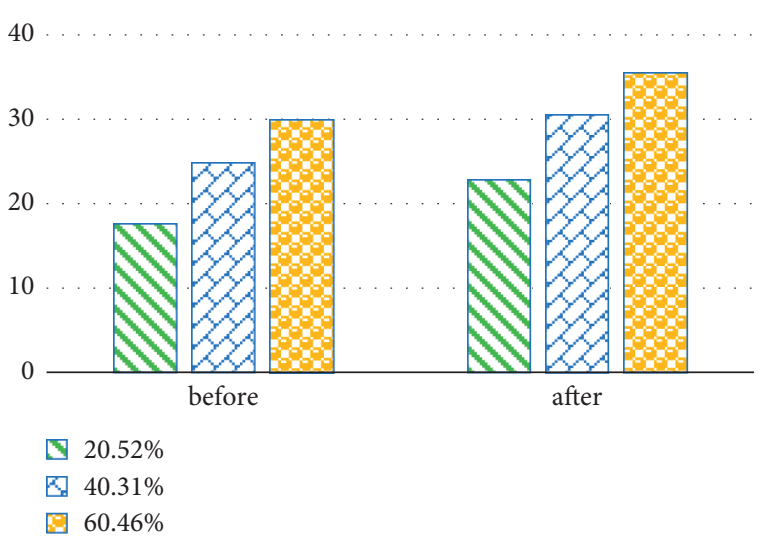

FIGURE 7: The signal-to-noise ratio of the reconstructed image of the knee joint MRI under the two-dimensional random sampling method.

image reconstruction was $24.72 \%$ and $30.4 \%$, respectively. When the sampling rate was $60.46 \%$, the signal-to-noise ratio before and after MRI image reconstruction was $29.84 \%$ and $35.42 \%$, respectively. Figure 8 shows that when the sampling rate of the radial sampling method was $21.91 \%$, the signal-to-noise ratio of MRI images before and after reconstruction was $12.94 \%$ and $13.02 \%$, respectively. When the sampling rate was $42.41 \%$, the signal-to-noise ratio before and after MRI image reconstruction was $20.34 \%$ and $24.17 \%$, respectively. When the sampling rate was $61.91 \%$, the signal-to-noise ratio before and after MRI image reconstruction was $27.7 \%$ and $32.23 \%$, respectively.

To more intuitively reflect the changes in the data, it was drawn into a graph, as shown in Figure 9 and 10.

By observing and comparing Figures 3 and 4, it was found that the quality (clarity, specificity, accuracy, etc.) of reconstructed MRI images based on CS theory was much higher than that before reconstruction (the images were fuzzy and the details were not prominent $)(P<0.05)$. By observing the data in Figures 5-8 and the change curves in Figures 9 and 10, it was found that, under the condition of almost the same sampling rate $(20 \%, 40 \%$, and $60 \%)$, the signal-to-noise ratio of reconstructed knee MRI images by random sampling was higher than that by radial sampling ( $9 \%, 6 \%$, and $3 \%$ higher, respectively). This verifies the fact that the diagnostic rate of MRI reconstructed images based on CS theory is better and also verifies the fact that random sampling contains more information than radial sampling.

\section{Discussion}

MRI is increasingly used in the diagnosis of bone and joint injuries, and the application value of MRI imaging features based on the reconstruction algorithm under the compressed sensing theory in diagnosing cartilage injuries is the current focus of imaging research. Moon et al. (2012) [28] diagnosed the damage of the epiphysis and epiphyseal cartilage by MRI and proved that MRI can confirm the intrachondral fracture and the damage of the proximal epiphyseal plate, which can show the bone marrow edema adjacent to the epiphyseal injury. There was a low signal on the T1WI image and a high signal on the FS-PDWI image. There was no statistically significant difference between the results and the histological results $(P>0.05)$. However, in the MRI imaging process, when the scanning time is too long, the utilization rate of the equipment will be affected, and the patient also has a certain physical and mental tolerance. These objective factors will cause artifacts. In other words, the imaging speed of MRI will cause the appearance of motion artifacts and contrast distortion, which will cause the MRI image to be blurred and some details cannot be highlighted. To avoid these drawbacks, compressed sensing was adopted in this research to reconstruct the MRI image, and it was found that its quality (clarity, specificity, accuracy, etc.) was much higher than before reconstruction. The signal-to-noise ratio data before and after the reconstruction was compared, and the difference has obvious statistical significance $(P<0.05)$. It showed that the MRI images under the reconstruction algorithm can effectively improve the diagnosis effect of knee joint epiphyseal injury. 


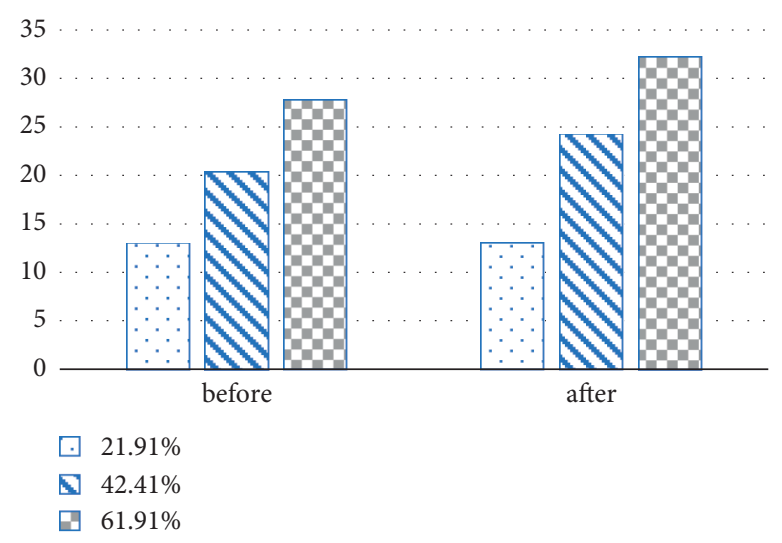

Figure 8: The signal-to-noise ratio of the reconstructed image of the knee joint MRI in the radial sampling mode.

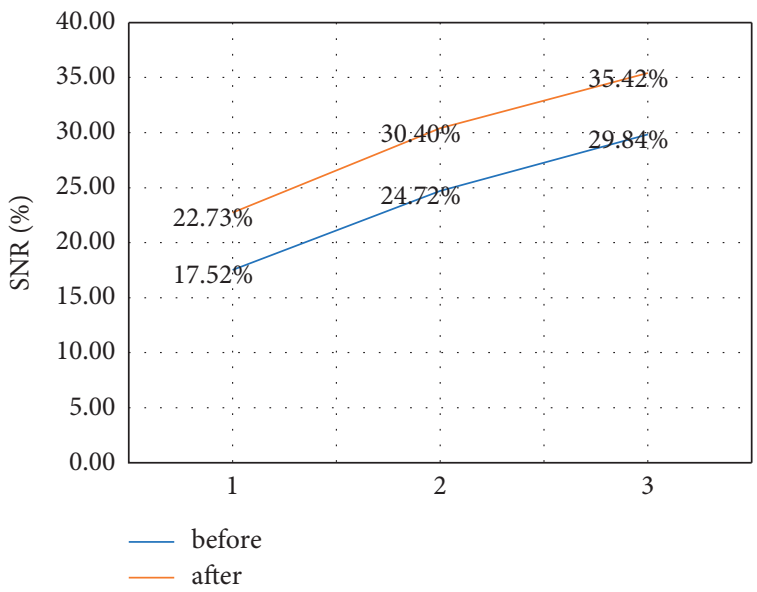

FIGURE 9: The change curve of the signal-to-noise ratio of the reconstructed image of the knee joint MRI in the two-dimensional random sampling mode $(1,2$, and 3 represent the sampling rates of $20.52 \%, 40.31 \%$, and $60.46 \%$, respectively).

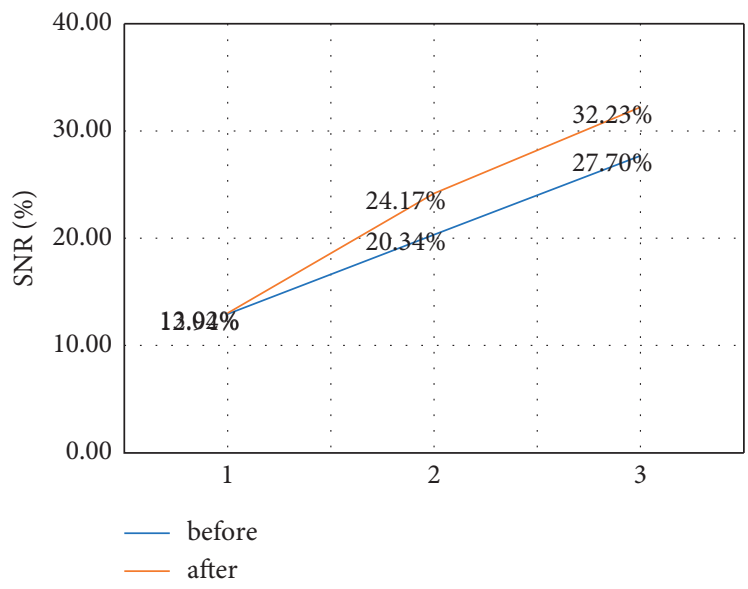

FIGURE 10: The change curve of the signal-to-noise ratio of the reconstructed image of the knee joint MRI in the radial sampling mode (1, 2 , and 3 represent sampling rates of $21.91 \%, 42.41 \%$, and $61.91 \%$, respectively). 
Zhao et al. (2020) [29] mentioned the high resolution of MRI in the study, which can clearly show the damage of the meniscus and ligament of the patient and can classify the damage. This was consistent with the opinion of this article on the MRI results of meniscal injury patients, that is, MRI can clearly diagnose the surrounding soft tissue injury, showing soft tissue swelling, soft tissue gaps are blurred, and the soft tissue was patchy with low T1WI and high FS-PDWI signals. On this basis, in this research experiment, two methods of two-dimensional random sampling and radial sampling were used to obtain magnetic resonance images, and different sampling rates were set to compare the reconstruction algorithms under compressed sensing. It was found that the two-dimensional random sampling had a higher signal-to-noise ratio than the MRI reconstructed image of the knee joint under the radial sampling method, and the difference was statistically significant $(P<0.05)$. This verified the fact that the diagnosis rate of MRI reconstructed images based on the compressed sensing theory was better and also verified the fact that random sampling contains more information than radial sampling. MRI imaging has high soft tissue resolution and can find tiny bone bridges, guide early clinical intervention, and improve the prognosis of patients [30-32]. So far, however, there are still relatively few studies on knee epiphyseal injuries in minors.

\section{Conclusion}

MRI images of children and adolescents with epiphyseal injury were analyzed and reconstructed based on CS theory. MRI images of $2 \mathrm{D}$ random sampling and radial sampling were compared before and after reconstruction. The MRI signs of knee epiphyseal injury included epiphyseal edema, epiphyseal plate widening, and periosteum reaction. Magnetic resonance imaging can also show concurrent injuries caused by cartilage injury, such as knee ligament, meniscus, and surrounding soft tissue injury. In the $2 \mathrm{D}$ random sampling mode, the reconstructed image has improved its clarity, specificity, and accuracy and has obvious effects and outstanding visual effects. This reconstruction algorithm has a great breakthrough in SNR and image details. Since the sample size of this study is relatively small and the follow-up time is short, the study will be further expanded in the future to verify this conclusion with more clinical trials.

\section{Data Availability}

The data used to support the findings of this study are available from the corresponding author upon request.

\section{Conflicts of Interest}

The authors declare that they have no conflicts of interest.

\section{References}

[1] P. T. Newton, L. Li, B. Zhou et al., "A radical switch in clonality reveals a stem cell niche in the epiphyseal growth plate," Nature, vol. 567, no. 7747, pp. 234-238, 2019 Mar.
[2] P. Persiani, F. M. Ranaldi, A. Formica et al., "Apophyseal and epiphyseal knee injuries in the adolescent athlete," La Clinica terapeutica, vol. 167, no. 6, pp. e155-e161, 2016.

[3] P. Gogna, S. Gaba, R. Mukhopadhyay, R. Rohilla, and A. Singh, "Neglected epiphyseal injuries of the distal end of the radius with ulnar impaction: analysis of distal osteotomy of both bones using a dorsal midline approach," Journal of Orthopaedics and Traumatology, vol. 18, no. 1, pp. 31-36, 2017.

[4] C. Guo, J. Lu, Z. Tian, W. Guo, and A. Darvishan, "Optimization of critical parameters of PEM fuel cell using TLBODE based on Elman neural network," Energy Conversion and Management, vol. 183, pp. 149-158, 2019.

[5] J. Bauer, I. Orendi, H. N. Ladenhauf, and T. Neubauer, "Knöcherne Knieverletzungen im Kindes- und Jugendalter," Unfallchirurg, Der, vol. 122, no. 1, pp. 6-16, 2019.

[6] C. P. Johnson, L. Wang, F. Tóth et al., "Quantitative susceptibility mapping detects neovascularization of the epiphyseal cartilage after ischemic injury in a piglet model of leggcalvé-perthes disease," Journal of Magnetic Resonance Imaging, vol. 50, no. 1, pp. 106-113, 2019.

[7] M. Hu, Y. Zhong, S. Xie, H. Lv, and Z. Lv, "Fuzzy system based medical image processing for brain disease prediction," Frontiers in Neuroscience, vol. 15, Article ID 714318, 2021.

[8] Q.-Q. Song, H. Liu, W. Lian et al., "The characteristics of positive and confusing hand X-ray signs in diagnosing Kashin-Beck Disease in children in China," Scientific Reports, vol. 8, no. 1, Article ID 3277, 2018.

[9] J. Hendrych, T. Pešl, and P. Havránek, "[Triplane fractures of the distal tibial epiphysis-contributions of CT scans to indication and planning of osteosynthesis]," Acta Chirurgiae Orthopaedicae et Traumatologiae Cechoslovaca, vol. 85, no. 5, pp. 336-342, 2018.

[10] F. Lecouvet, T. Van Haver, S. Acid et al., "Magnetic resonance imaging (MRI) of the knee: identification of difficult-to-diagnose meniscal lesions," Diagnostic and Interventional Imaging, vol. 99, no. 2, pp. 55-64, 2018.

[11] C. Zhang, S. Shen, W. Zhang et al., "Effect of long-term diving on the morphology and growth of the distal radial epiphyseal plate of young divers," Clinical Journal of Sport Medicine, vol. 29, no. 4, pp. 312-317, 2017 Jul.

[12] Y. Li, J. Zhao, Z. Lv, and J. Li, "Medical image fusion method by deep learning," International Journal of Cognitive Computing in Engineering, vol. 2, pp. 21-29, 2021.

[13] G. Dai, Z. He, and H. Sun, "Ultrasonic block compressed sensing imaging reconstruction algorithm based on wavelet sparse representation," Current Medical Imaging Formerly Current Medical Imaging Reviews, vol. 16, no. 3, pp. 262-272, 2020.

[14] T. Du, Y. Zhang, X. Shi, and S. Chen, "Multiple slice k-space deep learning for magnetic resonance imaging reconstruction," in Proceedings of the 2020 42nd Annual International Conference of the IEEE Engineering in Medicine \& Biology Society (EMBC), pp. 1564-1567, Montreal, Canada, 2020 July.

[15] J. Cao, S. Liu, H. Liu, and H. Lu, "CS-MRI reconstruction based on analysis dictionary learning and manifold structure regularization," Neural Networks, vol. 123, pp. 217-233, 2020.

[16] L. Gong, L. Duan, Y. Dai et al., "Locally adaptive total p-variation regularization for non-rigid image registration with sliding motion," IEEE Transactions on Biomedical Engineering, vol. 67, no. 9, pp. 2560-2571, 2020.

[17] C. He, F. Xu, Y. Liu, and J. Zheng, "A fast kernel extreme learning machine based on conjugate gradient," Network (Bristol, England), vol. 29, no. 1-4, pp. 70-80, 2018. 
[18] L. Zhu, H. Zhao, and H. Wang, "Partial differential equation modeling of rumor propagation in complex networks with higher order of organization," Chaos: An Interdisciplinary Journal of Nonlinear Science, vol. 29, no. 5, Article ID 053106, 2019.

[19] J. K. Pant and S. Krishnan, "Compressive sensing of electrocardiogram signals by promoting sparsity on the secondorder difference and by using dictionary learning," IEEE Transactions on Biomedical Circuits and Systems, vol. 8, no. 2, pp. 293-302, 2014.

[20] H. Liu, X. Gao, P. H. Schimpf, F. Yang, and S. Gao, "A recursive algorithm for the three-dimensional imaging of brain electric activity: shrinking LORETA-FOCUSS," IEEE Transactions on Biomedical Engineering, vol. 51, no. 10, pp. 17941802, 2004.

[21] Z. Lv, L. Qiao, Q. Wang, and F. Piccialli, “Advanced machinelearning methods for brain-computer interfacing," IEEE/ ACM Transactions on Computational Biology and Bioinformatics, vol. 18, no. 5, pp. 1688-1698, 2021.

[22] A. Majumdar, R. K. Ward, and T. Aboulnasr, "Non-convex algorithm for sparse and low-rank recovery: application to dynamic MRI reconstruction," Magnetic Resonance Imaging, vol. 31, no. 3, pp. 448-455, 2013.

[23] X.-J. Chen, C.-H. Luo, and M.-H. Chen, "Combination of "generalized Trotter operator splitting" and "quadratic adaptive algorithm" method for tradeoff among speedup, stability, and accuracy in the Markov chain model of sodium ion channels in the ventricular cell model," Medical, \& Biological Engineering \& Computing, vol. 58, no. 9, pp. 21312141, 2020.

[24] C.-H. Luo, X. J. Chen, X.-J. Chen, and M.-H. Chen, “Combination of multi-variable quadratic adaptive algorithm and hybrid operator splitting method for stability against acceleration in the Markov model of sodium ion channels in the ventricular cell model," Mathematical Biosciences and Engineering, vol. 17, no. 2, pp. 1808-1819, 2020.

[25] Q. Guo, Y. Teng, C. Tong, D. Li, and X. Wang, "[Brain functional network reconstruction based on compressed sensing and fast iterative shrinkage-thresholding algorithm]," Sheng Wu Yi Xue Gong Cheng Xue Za Zhi, vol. 37, no. 5, pp. 855-862, 2020.

[26] H. Wang, Y. Zhou, X. Wu, W. Wang, and Q. Yao, "Reconstruction of compressively sampled MR images based on a local shrinkage thresholding algorithm with curvelet transform," Medical, \& Biological Engineering \& Computing, vol. 57, no. 10, pp. 2145-2158, 2019.

[27] D. J. Cepela, J. P. Tartaglione, T. P. Dooley, and P. N. Patel, "Classifications in brief: salter-harris classification of pediatric physeal fractures," Clinical Orthopaedics and Related Research, vol. 474, no. 11, pp. 2531-2537, 2016 Nov.

[28] S. G. Moon, H. S. Kang, S.-H. Hong, N. R. Kim, J. W. Lee, and S. D. Lim, "Chronologic change in the growth plate after radiofrequency-induced thermal injury: MRI-histologic correlation," American Journal of Roentgenology, vol. 198, no. 2, pp. W163-W172, 2012.

[29] M. Zhao, Y. Zhou, J. Chang et al., "The accuracy of MRI in the diagnosis of anterior cruciate ligament injury," Annals of Translational Medicine, vol. 8, no. 24, p. 1657, 2020.

[30] D. Abbasi, M. M. May, E. J. Wall, G. Chan, and S. N. Parikh, "MRI findings in adolescent patients with acute traumatic knee hemarthrosis," Journal of Pediatric Orthopaedics, vol. 32, no. 8, pp. 760-764, 2012.

[31] R. J. Bai, H. L. Zhan, Y. Liu et al., "[Focal periphyseal edema zone on MRI and clinical significance of the adolescent knee]," Zhonghua Yixue Zazhi, vol. 96, no. 25, pp. 1965-1970, 2016.

[32] H. N. Ladenhauf, K. J. Jones, H. G. Potter, J. T. Nguyen, and D. W. Green, "Understanding the undulating pattern of the distal femoral growth plate: implications for surgical procedures involving the pediatric knee: a descriptive MRI study," The Knee, vol. 27, no. 2, pp. 315-323, 2020. 